\title{
AN INTRODUCTION TO MESIC NUCLEI
}

\section{COLIN WiLKIN}

Physics and Astronomy Department, University College London

Gower Street, London, WC1E 6BT, UK

(Received September 21, 2015)

There is much speculation and a modest amount of evidence that certain mesons might form quasi-bound states with nuclei to produce really exotic states of matter. For this to be a practical possibility, the interaction between the meson and nucleons at low energies must be strong and attractive and the production rates "healthy". The conditions for this are surveyed for the $\bar{K}, \eta, \omega, \eta^{\prime}$, and $\phi$ mesons. How this might lead to quasi-bound states is then discussed in a few typical cases. Though some interesting effects have been seen in above-threshold data, the search for experimental signals for these exotic states with different mesons in bound state regions has generally been rather frustrating, with positive claims only being made for the $\eta$ and the $K^{-}$.

DOI:10.5506/APhysPolB.47.249

\section{Introduction}

Though the field had been around for more than 20 years, in 2010 the APS finally recognised that there was a subject called "mesic nuclei", as indicated by the PACS number of the article.

The subject is of interest for a variety of reasons:

- It allows one to investigate the interaction of unstable particles with nucleons and nuclei.

- If such states existed, they would represent exotic nuclear matter with several hundred $\mathrm{MeV}$ of excitation energy.

- If one can produce a ${ }_{\eta}^{3} \mathrm{He}$ through $d p \rightarrow{ }_{\eta}^{3} \mathrm{He}$, this will contribute a small amount to $d p \rightarrow{ }_{\eta}^{3} \mathrm{He} \rightarrow d p$, i.e., deuteron-proton elastic scattering. 


\section{Near-threshold production of mesons in elementary reactions}

There is ample evidence from many sources that the s-wave $\pi^{0} p$ interaction is very weak and it stays weak until the p-wave $\Delta(1232)$ is approached. Nobody has therefore serious hopes of exotic nuclei involving pions and so we turn immediately to heavier mesons.

\section{1. $\eta$ and $\eta^{\prime}$ production}

If one only includes the S-wave and its final state interaction (FSI) in the $p p$ system in the simplest possible approximation [1], one expects the $p p \rightarrow p p \eta$ total cross section to vary as

$$
\sigma_{\mathrm{T}}(p p \rightarrow p p \eta)=C\left(\frac{Q}{\varepsilon}\right)^{2} /(1+\sqrt{1+Q / \varepsilon})^{2},
$$

where the excess energy $Q=W-\left(2 m_{p}+m_{\eta}\right) c^{2}$, with $W$ being the total c.m. energy. The constant $C$ depends upon the reaction mechanism and can be adjusted to fit the data.

Since the Coulomb repulsion has here been neglected, there is some ambiguity in the value to take for the pole position $\varepsilon$. The best fit to the analogous $\eta^{\prime}$ production data [2-7] was achieved with $\varepsilon=0.75_{-0.15}^{+0.20} \mathrm{MeV}$ [7], which is quite consistent with the original theoretical assumptions [1]. The resulting curves for $\eta$ and $\eta^{\prime}$ production are compared to experimental data in Fig. 1.

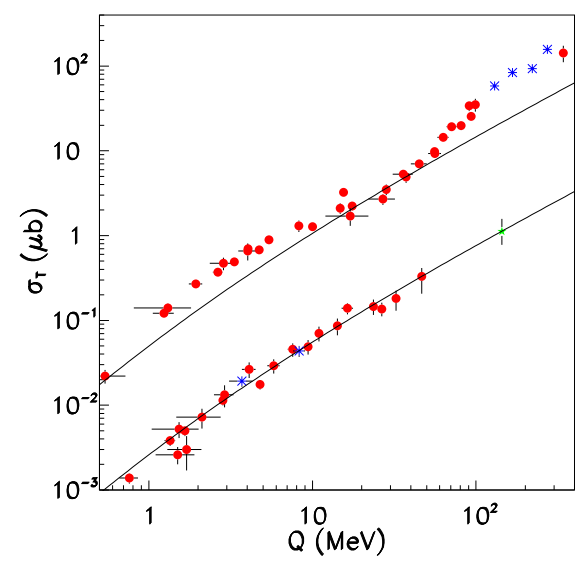

Fig. 1. Total cross sections for $p p \rightarrow p p \eta$ (upper points) and $p p \rightarrow p p \eta^{\prime}$ (lower points). The $\eta$ data are taken from Refs. [2, 3, 8-12] (closed red circles), and [13] (blue crosses) and the $\eta^{\prime}$ data from Ref. [2, 3] (blue crosses), [4] (green star), and [5-7] (closed red circles). The solid curves are arbitrarily scaled $p p$ FSI predictions of Eq. (1). 
In the $\eta$ case, there are large deviations from the curve at low $Q$ that may be ascribed to a strong $\eta$-nucleon FSI. The deviations at large $Q$ are likely to originate from $\mathrm{P}$ or higher waves in the final $p p$ system. The situation is very different for $\eta^{\prime}$ production, where there is no sign of any FSI in the near-threshold data. The COSY-11 Collaboration has put limits on the $\eta^{\prime} p$ scattering length but one can see immediately from the figure that the FSI is very much stronger for $\eta$ production. One cannot make statements regarding the influence of $\mathrm{P}$-waves for the $\eta^{\prime}$ at large $Q$ due to the lack of data there. The other point that is worth noting is the factor of about twenty between $\eta$ and $\eta^{\prime}$ production cross sections.

\section{2. $\omega$ production}

The situation is much less certain for $\omega$ production. The $p p \rightarrow p p \omega$ total cross section is of the same order of magnitude as that for $\eta$ production but, because of its natural width $\Gamma_{\omega} \approx 8.5 \mathrm{MeV} / c^{2}$, the missing-mass peak is generally less narrow. The comparison of the data in Fig. 2 with the predictions of Eq. (1) looks very similar to that of the $\eta$ in Fig. 1. The strong deviations seen at high $Q$ are likely to originate from contributions of $\mathrm{P}$ and higher waves in the final $p p$ system. At low $Q$, the points also lie above the (dashed) $p p$ FSI curve. This is (probably) not due to any attraction between the $\omega$ and a proton but rather it is an effect of the natural $\omega$ width. Even when the nominal $Q$ is negative, one can still produce the low mass tail of the $\omega$. The solid (red) curve tries to take this into account [14].

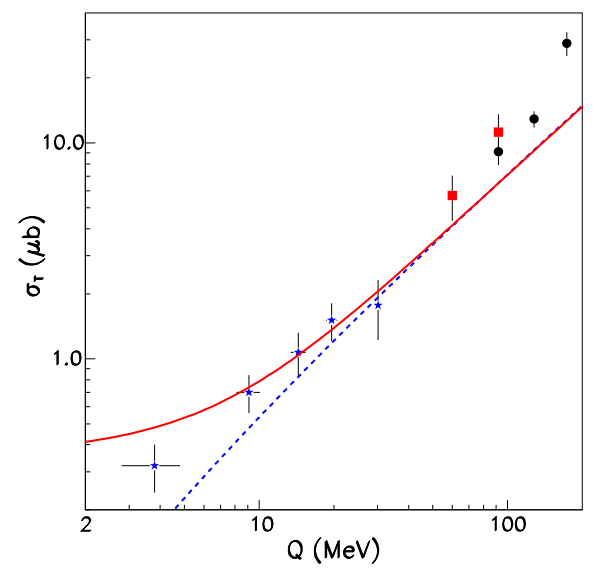

Fig. 2. Total cross sections for $p p \rightarrow p p \omega$ in terms of the nominal value of $Q$, i.e., neglecting the $\omega$ width. The data are taken from Refs. [14] (blue crosses), [15] (red squares), and [16] (black circles). The dashed (blue) curve is an arbitrarily scaled $p p$ FSI prediction of Eq. (1), whereas the solid (red) one has been smeared over the $\omega$ width. 
Since it is hard to draw conclusions on the $\omega p$ FSI from the $p p \rightarrow p p \omega$ data of Fig. 2, let us turn instead to the new near-threshold data on $\gamma p \rightarrow$ $p \omega[17]^{1}$. Smearing over the $\omega$ decay width is less critical here because the unsmeared cross section rises far more rapidly from the threshold than it does for $p p \rightarrow p p \omega$. The threshold value derived from the fit shown in Fig. 3 is $\sigma_{\mathrm{T}} / k \approx 0.044 \mu \mathrm{b} / \mathrm{MeV} / c$. In the vector-dominance model, the photon reactions are related to ones driven by incident $\rho, \omega$, and $\phi$ vector mesons, from which one can get an estimate of the $\omega p$ scattering length, $\left|a_{\omega p}\right|=(0.82 \pm 0.03) \mathrm{fm}$. However, one does not really know how much of this is due to the $\rho$-meson or the Born term.

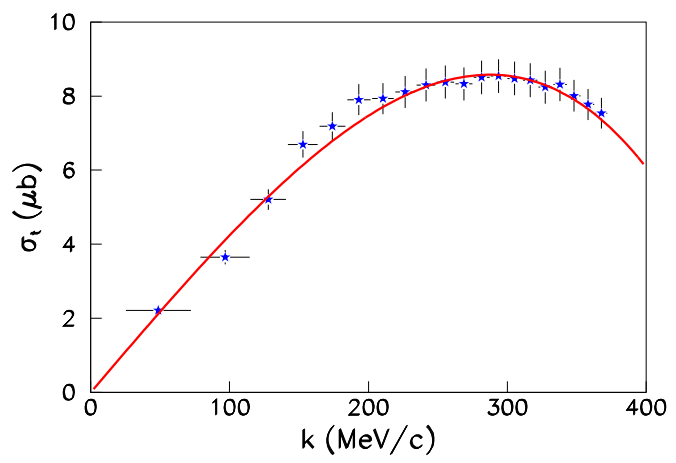

Fig. 3. The total cross section for $\gamma p \rightarrow p \omega$ as a function of the nominal (zero $\omega$ width) value $k$ of the final c.m. momentum [17]. The data are compared to a fit of the form $\sigma_{\mathrm{T}}=a_{1} k+a_{3} k^{3}+a_{5} k^{5}$.

Theoretical models give typically much smaller values of the scattering length, e.g., $a_{\omega p}=(-0.026+i 0.28)$ fm from the coupled-channel analysis of $\omega$ production in $\gamma N$ and $\pi N$ interactions [19], but there are lots of other models on the market.

\section{3. $\phi$ production}

The available data on the $p p \rightarrow p p \phi$ total cross section are shown in Fig. 4, along with the curve corresponding to the simple $p p$ FSI approach of Eq. (1). The good agreement with the curve may be completely fortuitous because the data show that many partial waves must be present at the higher excess energies. It should, however, be noted that the cross section is about a factor of 30 lower than that for $\omega$ production. There is a lack of data at small $Q$ and so it is not clear from these results whether the s-wave $\phi p$ interaction is attractive or not.

\footnotetext{
${ }^{1}$ New data on this reaction have appeared post-symposium from CBELSA [18].
} 
It may seem surprising that the energy dependence of $\phi$ production is better measured in the $p n \rightarrow d \phi$ reaction [22]. The message is, however, similar in that the total cross section behaves like $\sqrt{Q}$, i.e., like phase space with no sign of any $\phi$ attraction to the deuteron at low energy. One should, nevertheless, bear in mind that the $\phi$ decay distribution shows higher partial waves above about $40 \mathrm{MeV}$.

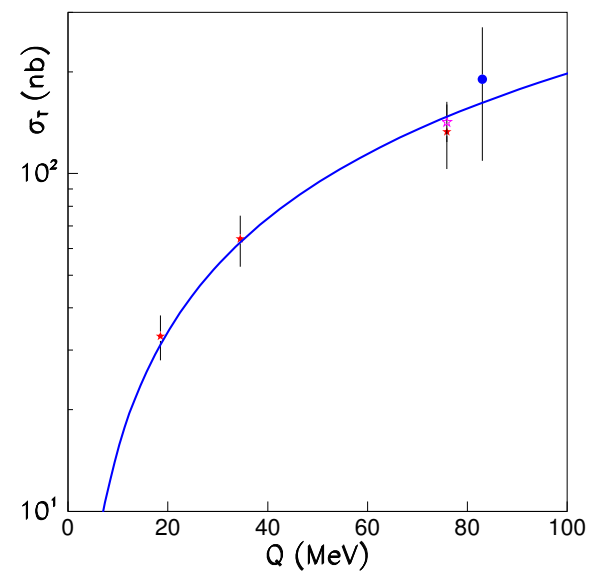

Fig. 4. The total cross section for $p p \rightarrow p p \phi$ measured at ANKE (red stars) [20] and DISTO (blue circle) [21]. The curve is the simple $p p$ FSI parameterisation of Eq. (1). For this range of $Q$, the smearing over the $\phi$ width is not important.

\section{4. $K^{-}$production}

Due to strangeness conservation, a $K^{-} p p$ or $K^{0} p p$ system cannot be produced in isolation in $p p$ collisions. The best that can be done is to look at kaon pair production, $p p \rightarrow K^{+} K^{-} p p$, and compare the $K^{+} p p$ and $K^{-} p p$ distributions. The $K^{+}$is believed to be weakly interacting with nucleons and the force may even be slightly repulsive!

Several experiments have shown that the $K^{-}$is strongly attracted to one or both protons in the $p p \rightarrow p p K^{+} K^{-}$reaction. To put this on a quantitative basis, define cross section ratios in terms of the $K^{ \pm} p$ and $K^{ \pm} p p$ invariant masses:

$$
R_{K p}=\frac{d \sigma / d M_{K^{-}}}{d \sigma / d M_{K^{+} p}}, \quad R_{K p p}=\frac{d \sigma / d M_{K^{-} p p}}{d \sigma / d M_{K^{+} p p}} .
$$

The distributions in $R_{K p}$ and $R_{K p p}$ obtained in an experiment below the $\phi$ threshold [23] are both shown in Fig 5 . The data are well-described with an effective scattering length of $a_{K^{-} p}=2.45 \mathrm{fm}$. Although $a_{K^{-}}$was taken to be purely imaginary, the data are not very sensitive to the phase. 

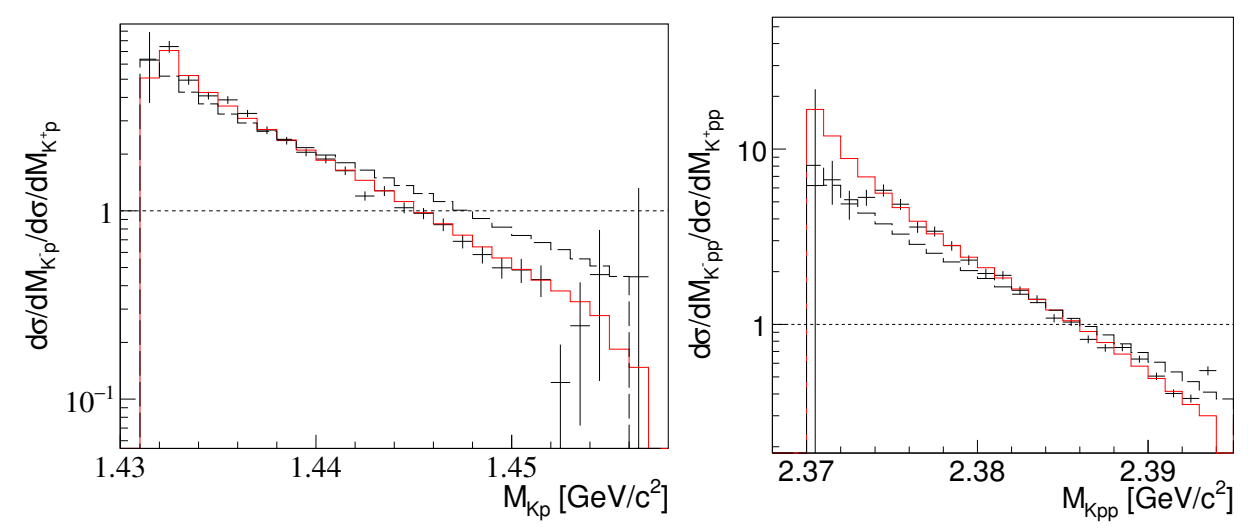

Fig. 5. The measured ratios $R_{K p}$ and $R_{K p p}$ at $Q=24 \mathrm{MeV}$ [23]. The solid (red) and broken black histograms represent estimations that take into account $K^{-} p$, $p p$ and $K^{+} K^{-}$final state interactions with $a_{K^{-} p}=2.45 i \mathrm{fm}$ and $a_{K^{-} p}=1.5 i \mathrm{fm}$, respectively.

\subsection{Summary of information from semi-inclusive measurements}

Both the $\eta$ and the $K^{-}$seem to be strongly attracted to protons at low energy. This is not a complete surprise because there are s-wave resonances sitting at (and overlapping with) the $\eta p$ and $K^{-} p$ thresholds. There are thus strong s-wave couplings for $N^{*}(1535): \eta p$ and $\Lambda(1405): K^{-} p$.

Since the $\eta$ is isoscalar, this means that the meson is also attracted to neutrons. However, data on $p n \rightarrow d K^{+} K^{-}$[24] can be interpreted as suggesting that the $K^{-}$attraction to neutrons is weaker than to protons, probably because there is no $I=1$ s-wave hyperon resonance near the $K^{-} n$ threshold. There is no firm evidence for strong $\eta^{\prime}, \omega$, or $\phi$ attraction to nucleons at low energies but the $\omega$ case is complicated by the decay width $\Gamma_{\omega} \approx 8.5 \mathrm{MeV} / c^{2}$.

\subsection{Information from inclusive experiments}

Cross sections for inclusive photoproduction of a meson from a nucleus with mass number $A$ are often fitted with $\sigma \sim A^{\alpha}$. If $\alpha$ is close to unity, the whole nucleus is participating and the meson interaction is weak. If $\alpha$ approaches $2 / 3$, only the back surface is contributing and the interaction is very strong. Data in Fig. 6 show that the nucleus is fairly transparent to low energy pions, but the picture changes when the $\Delta(1232)$ is reached. On the other hand, the $\eta$ is strongly absorbed at all the energies shown.

The $\eta^{\prime}$ is an intermediate case but the $\omega$ also looks to be strongly absorbed. However, only in the $\pi$ or possibly the $\eta$ case do the data extend into the near-threshold s-wave domain. There are also complications from 
two-step processes, e.g., $\gamma N \rightarrow \pi N$ followed by $\pi N \rightarrow \omega N$. These might actually be kinematically favoured because they share the momentum transfer better.

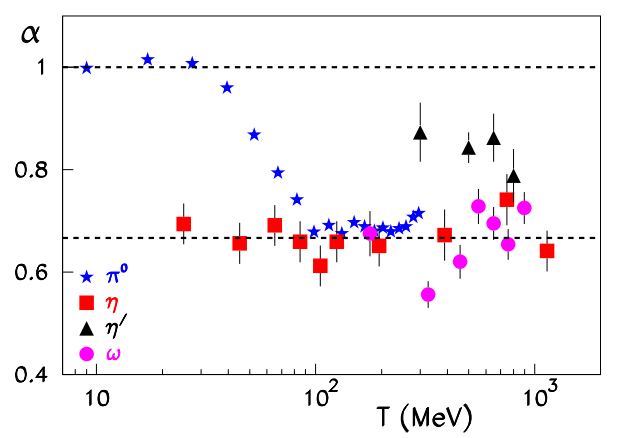

Fig. 6. Scaling parameter $\alpha$ as a function of meson kinetic energy $T$ for $\pi^{0}$ [25], $\eta[26,27], \eta^{\prime}$ [28], and $\omega$ mesons [29]. Pion data were not plotted above $300 \mathrm{MeV}$.

The $A^{\alpha}$ parameterisation is very close to that of the nuclear transparency approach, where one compares the production on a nucleus to that on a reference nucleus, which is invariably carbon, to form the ratio

$$
R=\left(\frac{12}{A}\right) \frac{\sigma_{A}}{\sigma_{\mathrm{C}}} .
$$

There are measurements of $R$ also in proton-nucleus collisions, e.g., in the production of the $\phi$ meson [30]. However, these are all for $T_{\phi}>160 \mathrm{MeV}$ and have little relevance for the mesic nucleus question.

All the models used to analyse such data have large contributions from two-step processes involving, perhaps, intermediate $\pi$ or $\omega$ mesons. Hence the interpretation depends on the models used and it is difficult to be sure from these how absorptive the s-wave $\phi p$ interaction really is. Nevertheless, this is one of the few ways of getting some information regarding the imaginary part of the potential between the meson and the nucleus.

It has been suggested that the energy dependence of, say, $\gamma A \rightarrow \eta^{\prime} A^{*}$ in and below the free nucleon threshold should be sensitive to the $\eta^{\prime} A^{*}$ potential [31]. There will always be production below this threshold due, e.g., to the Fermi motion. But, if the $\eta^{\prime}$ is attracted to the nucleons, that effectively reduces the total mass of a cluster and so this might be produced at lower photon energies. A lot of corrections are included in the modelling but it is not evident that all the uncertainties arising from the assumptions in the model are taken into account. The distinction between the production on nucleon clusters and two-step processes is not always clear and two-step effects depend critically upon the particular meson produced. 


\section{Production rates}

In order to form a mesic nucleus, a good production rate is needed as well as a strong attraction of the meson to a nucleus. Though some information on relative production rates is contained in the $p p \rightarrow p p X$ data, the $p d \rightarrow{ }^{3} \mathrm{He} X$ rates might be more informative. Define the average amplitude squared by

$$
\left|f\left(p d \rightarrow{ }^{3} \mathrm{He} X\right)\right|^{2}=\frac{p_{d}}{p_{X}} \frac{d \sigma}{d \Omega}\left(p d \rightarrow{ }^{3} \mathrm{He} X\right) .
$$

Close to their thresholds $\left|f\left(p d \rightarrow{ }^{3} \mathrm{He} \eta\right)\right|^{2} \approx 2500 \mathrm{nb} / \mathrm{sr},\left|f\left(p d \rightarrow{ }^{3} \mathrm{He} \omega\right)\right|^{2} \approx$ $30 \mathrm{nb} / \mathrm{sr},\left|f\left(p d \rightarrow{ }^{3} \mathrm{He} \eta^{\prime}\right)\right|^{2} \approx 0.9 \mathrm{nb} / \mathrm{sr},\left|f\left(p d \rightarrow{ }^{3} \mathrm{He} \phi\right)\right|^{2} \approx 2.3 \mathrm{nb} / \mathrm{sr}$.

Thus $\eta^{\prime}$ production is more than three orders of magnitude weaker than for the $\eta$, compared to a mere factor of twenty in $p p$ collisions. The smallness of $\eta^{\prime}$ production seems to be confirmed by unpublished COSY-WASA data [32]. If the acceptance of WASA is estimated on the basis of phase space, then $\left|f\left(p d \rightarrow{ }^{3} \mathrm{He} \omega\right)\right|^{2} \approx 11 \mathrm{nb} / \mathrm{sr}$ at $Q=240 \mathrm{MeV}$ and $\mid f(p d \rightarrow$ $\left.{ }^{3} \mathrm{He} \eta^{\prime}\right)\left.\right|^{2} \approx 0.6 \mathrm{nb} / \mathrm{sr}$ at $Q=64 \mathrm{MeV}$. Of course, these are rough estimates but one would not be saved by a factor of two in the $\eta^{\prime}$ case.

\section{Estimation of binding energies}

Liu and Haider [33] started the whole bound $\eta$-mesic business through their estimates of binding within single-channel potential models, where $V_{\eta A} \propto f_{\eta N} \rho(r)$, with $\rho(r)$ being the nuclear density and $f_{\eta N}$ the $\eta$-nucleon elastic scattering amplitude. This leaves several problems:

- One does not know what to assume for $f_{\eta N}$.

- Due to the $N^{*}(1535)$ resonance, the potential is likely to have a strong energy dependence. How can this be taken into account? Which energy should one choose?

- It seems as though there may be nearby poles in the $\eta^{3} \mathrm{He}$ and $\eta^{4} \mathrm{He}$ systems. Who would trust the predictions of a one-particle optical potential for such light nuclei?

- Use of such potentials suggest that the binding energy of a meson to the ground state of a nucleus is likely to be similar in magnitude to that of one of its excited states, so that

$$
M\left({ }_{\eta}^{12} \mathrm{C}\left(2^{+}\right)\right)-M\left({ }_{\eta}^{12} \mathrm{C}\left(0^{+}\right)\right) \approx 4.4 \mathrm{MeV} .
$$

This means that one would need very favourable kinematic conditions if the mesic nuclear widths were as large as the nuclear level spacing. 
It should be noted that the situation is not saved by neglecting states above the break-up threshold because the $\eta$ could just as well stick to one of the nuclear residues.

A Japanese group [34] has made estimates of the binding of an $\eta^{\prime}$ to ${ }^{12} \mathrm{C}$ in an optical potential approach for a variety of potential strengths. One could argue that their potential is too attractive, given what we know about the $\eta^{\prime}$-nucleon interaction, but the widths that they predict are large compared to the nuclear level spacing. The nuclear excited states problem is therefore likely to hinder most $\eta^{\prime}$ mesic nuclei searches.

\section{Mesic nucleus experiments}

There are two very different methods to search for mesic nuclei:

1. Measure meson production at a few energies just above threshold and attempt to extrapolate to below threshold, where a quasi-bound nucleus may reside. This approach does overcome the very serious background problem but it could only work if the mesic nucleus were lightly bound. Even more troublesome is the fact that above-threshold experiments can never distinguish between bound and virtual (antibound) states. This is just like asking if one can deduce that the ${ }^{3} S_{1} n p$ has a bound state (deuteron) but the ${ }^{1} S_{0}$ has none if one only looks above the $n p$ threshold. A typical (i.e., best) example is $d p \rightarrow{ }^{3} \mathrm{He} \eta$.

2. Look directly in the bound state region. By definition, the meson cannot emerge and the background could be overwhelming unless one could identify the quasi-free decay of the meson. But this is the only way to be $100 \%$ sure that one has a quasi-bound state. One tries to suppress the background by choosing "favourable" kinematics. A typical example of this in the $\eta$ case is ${ }_{\eta}^{3} \mathrm{He} \rightarrow \pi^{0} p X$, where the $\pi^{0}$ and $p$ come out back-to-back in the overall c.m. frame.

\subsection{An above-threshold search}

There are a lot of data on $d p \rightarrow{ }^{3} \mathrm{He} \eta$. The total cross section jumps to its plateau value within about $0.5 \mathrm{MeV}$ of the threshold. The jump is even sharper if the beam momentum distribution is taken into account [35]. There is a pole in the $\eta^{3}$ He scattering amplitude at $p_{\eta}=(-5 \pm 7) \pm i(19 \pm 3) \mathrm{MeV} / c$, i.e., at $Q=(-0.30 \pm 0.15) \pm i(0.21 \pm 0.06) \mathrm{MeV}$. Of course, the real part can even vanish by chance, but why is the imaginary part so small?

If the pole is due to the $\eta^{3} \mathrm{He}$ FSI, it should be present for all entrance channels. A big near-threshold jump is seen in $\gamma^{3} \mathrm{He} \rightarrow \eta^{3} \mathrm{He}$ but the resolution is not as good as in the hadronic experiments [36]. The best proof of the 
FSI hypothesis is the deuteron tensor analysing power $T_{20}$ in $\overrightarrow{d p} \rightarrow{ }^{3} \mathrm{He} \eta$, which is sensitive to the spin- $3 / 2 /$ spin- $1 / 2$ ratio in the initial $d p$ state. The value of $T_{20}$ is effectively constant near threshold [37], despite the cross section jumping around.

\subsection{A sub-threshold search}

Most sub-threshold (i.e. direct) searches have given disappointing results. In the case of $\gamma^{3} \mathrm{He} \rightarrow \pi^{0} p X$, if this passes through an ${ }_{\eta}^{3} \mathrm{He}$ mesic nucleus, there could be a peak in the energy distribution of back-to-back $\pi^{0} p$ pairs in the overall c.m. frame. The first MAMI experiment [38] found evidence for such a peak by subtracting data in one angular bin from another. Later MAMI data [36] confirmed the existence of peak but showed that the interpretation was very "suspect". The energy dependence showed lots of structure but this seemed to evolve smoothly with $\pi^{0} p$ opening angle. There was no sign of any mesic nucleus decay.

\subsection{Quasi-bound $K^{-}$pp systems}

The $K^{+} / K^{-}$distortions in the COSY $p p \rightarrow K^{+} K^{-} p p$ data $[20,23]$ seem to be driven mainly by $p p \rightarrow K^{+} p \Lambda(1405)$ and the decay of the tail of $\Lambda(1405) \rightarrow K^{-} p$ [39]. This might be an indication for a lightly bound $K^{-} p p$, or $\Lambda(1405) p$, system, which could correspond to an $S=-1, B=2$ mesic nucleus. Such a mesic nucleus could decay via $\Sigma^{0} \pi^{0} p$, but counting rates for $p p \rightarrow K^{+} \Sigma^{0} \pi^{0} p$ are not very high and the acceptance of the available spectrometers for multiparticle final states are rather low!

There is a severe lack of experimental data for other nuclei. COSY studied $p d \rightarrow{ }^{3} \mathrm{He} K^{+} K^{-}$, but there was no magnetic field for the kaon detection so that they could only produce average $K^{ \pm 3} \mathrm{He}$ distributions, which are little sensitive to the $K^{-3} \mathrm{He}$ interaction [40].

But are there systems that are bound so deeply that they can only decay via hyperon production? The subject is VERY controversial!

\subsection{Deeply bound $K^{-}$pp systems}

Yamazaki et al. [41] took the $2.85 \mathrm{GeV}$ DISTO data [42] and divided by a phase-space distribution that was passed through the DISTO analysis program. They generated a $\Lambda p$ invariant-mass peak with $M \approx 2267 \mathrm{MeV} / c^{2}$ and $\Gamma \approx 118 \mathrm{MeV} / c^{2}$. This could be interpreted as a $\Lambda(1405) p$ bound state, which they called $X(2267)$. However, there was no sign of such a state in the $2.5 \mathrm{GeV}$ DISTO data.

The procedure was studied in detail by Epple and Fabbietti [43], who analysed the $3.5 \mathrm{GeV}$ HADES data [44]. They found that the generated shape depended on the cuts imposed because the data just did not look 
like phase space. They also questioned Yamazaki's estimate of the energy dependence of $p p \rightarrow p \Lambda(1405)$, which was supposed to be the doorway to his $X(2267)$ state. Their criticism is described in detail in the Fabbietti contribution to this workshop, to which the reader is referred [43].

\section{REFERENCES}

[1] G. Fäldt, C. Wilkin, Phys. Lett. B 382, 209 (1996).

[2] A.M. Bergdolt et al., Phys. Rev. D 48, R2969 (1993).

[3] F. Hibou et al., Phys. Lett. B 438, 41 (1998).

[4] F. Balestra et al., Phys. Lett. B 491, 29 (2000).

[5] P. Moskal et al., Phys. Rev. Lett. 80, 3202 (1998).

[6] P. Moskal et al., Phys. Lett. B 474, 416 (2000).

[7] E. Czerwiński et al., Phys. Rev. Lett. 113, 062004 (2014).

[8] E. Chiavassa et al., Phys. Lett. B 322, 270 (1994).

[9] H. Calén et al., Phys. Lett. B 366, 39 (1996).

[10] J. Smyrski et al., Phys. Lett. B 474, 182 (2000).

[11] P. Moskal et al., Phys. Rev. C 69, 025203 (2004).

[12] P. Moskal et al., Eur. Phys. J. A 43, 131 (2010).

[13] N. de Marco, private communication, 2001.

[14] F. Hibou et al., Phys. Rev. Lett. 83, 492 (1999).

[15] S. Barsov et al., Eur. Phys. J. A 29, 95 (2007).

[16] M. Abdel-Bary et al., Eur. Phys. J. A 44, 7 (2010).

[17] I.I. Strakovsky et al., Phys. Rev. C 91, 045207 (2015).

[18] A. Wilson et al., Phys. Lett. B 749, 407 (2015).

[19] V. Shklyar et al., Phys. Rev. C 71, 055206 (2005).

[20] M. Hartmann et al., Phys. Rev. Lett. 96, 242301 (2006); Q.J. Ye et al., Phys. Rev. C 85, 035211 (2012).

[21] F. Balestra et al., Phys. Rev. C 63, 024004 (2001).

[22] Y. Maeda et al., Phys. Rev. Lett. 97, 142301 (2006).

[23] Q.J. Ye et al., Phys. Rev. C 87, 065203 (2013).

[24] Y. Maeda et al., Phys. Rev. C 79, 018201 (2009).

[25] B. Krusche et al., Eur. Phys. J. A 22, 277 (2004).

[26] M. Röbig-Landau et al., Phys. Lett. B 373, 45 (1996).

[27] T. Mertens et al., Eur. Phys. J. A 38, 195 (2008).

[28] M. Nanova et al., Phys. Lett. B 710, 600 (2012).

[29] M. Kotulla et al., Phys. Rev. Lett. 100, 192302 (2008).

[30] A. Polyanskiy et al., Phys. Lett. B 695, 74 (2011). 
[31] M. Nanova et al., Phys. Lett. B 727, 417 (2013).

[32] M. Wolke, J. Złomanczuk, private communication, 2013.

[33] Q. Haider, L. C. Liu, Phys. Lett. B 172, 257 (1986).

[34] D. Jido, H. Nagahiro, S. Hirenzaki, Phys. Rev. C 85, 032201 (2012).

[35] T. Mersmann et al., Phys. Rev. Lett. 98, 242301 (2007).

[36] F. Pheron et al., Phys. Lett. B 709, 21 (2012).

[37] M. Papenbrock et al., Phys. Lett. B 734, 333 (2014).

[38] M. Pfeiffer et al., Phys. Rev. Lett. 92, 252001 (2004).

[39] J.-J. Xie, C. Wilkin, Phys. Rev. C 82, 025210 (2010).

[40] F. Bellemann et al., Phys. Rev. C 75, 015204 (2007).

[41] T. Yamazaki et al., Phys. Rev. Lett. 104, 132502 (2010).

[42] M. Maggiora et al., Nucl. Phys. A 691, 329c (2001).

[43] E. Epple, L. Fabbietti, Phys. Rev. C 92, 044002 (2015) [arXiv: 1504.02060 [nucl-ex]]; talk presented at the Symposium on Fundamental and Applied Subatomic Physics, Kraków, Poland, June 7-12, 2015.

[44] G. Agakishiev et al., Phys. Lett. B 742, 242 (2015). 